\title{
A Summary of Team MIT's Approach to the Virtual Robotics Challenge
}

\author{
R. Tedrake, M. Fallon, S. Karumanchi, S. Kuindersma, M. Antone, T. Schneider, T. Howard, M. Walter, \\ H. Dai, R. Deits, M. Fleder, D. Fourie, R. Hammoud, S. Hemachandra, P. Ilardi, C. Pérez-D’Arpino, S. Pillai, \\ A. Valenzuela, C. Cantu, C. Dolan, I. Evans, S. Jorgensen, J. Kristeller, J. A. Shah, K. Iagnemma, S. Teller
}

The attached video describes the system developed by researchers from MIT for the Defense Advanced Research Projects Agency's (DARPA) Virtual Robotics Challenge (VRC), held in June 2013. The VRC was the first competition in the DARPA Robotics Challenge (DRC), a program that aims to "develop ground robotic capabilities to execute complex tasks in dangerous, degraded, human-engineered environments" [1]. The VRC required teams to guide a model of Boston Dynamics' humanoid robot, Atlas, through driving, walking, and manipulation tasks in simulation.

Team MIT's approach centered around teleautomation, in which an operator provided high-level instructions to be carried out autonomously by the robot. The resulting system comprised a 3D user interface coupled with perception, planning, control, and networking modules. The system's perception and planning abilities were assisted by the human operator(s) as needed.

Team MIT's user interface, the Viewer, provided the operator with a unified representation of all available information. A 3D rendering of the robot depicted its most recently estimated body state with respect to the surrounding environment, represented by point clouds and texture-mapped meshes as sensed by on-board LIDAR and fused over time.

Inertial and kinematic measurements were combined to provide accurate estimates of the robot's body state. The pose of the robot's pelvis was estimated by freely propagating inertial acceleration and rotation rate measurements. Joint angle measurements were combined with the kinematic robot model to propagate special footstep representations in the local navigational frame. Discrepancies between inertial and kinematic estimates were used in a feedback process to correct inertial sensor and navigational state errors.

To overcome the uplink restrictions of the VRC, Team MIT's networking module employed an informationtheoretic coding algorithm to compress plans prior to sending them to the robot. This encoder, based on the Goby Project [2], allowed for compression ratios of over 30:1.

The planning and control infrastructure consisted of two modes: manipulation and walking. The operator initiated manipulation planning by requesting a grasp for a specified object. A grasp optimizer then suggested an appropriate grasp location for the indicated hand. The operator could then request manipulation plans to reach for, grasp, or move the object. Requests were transmitted to a whole-body planner that computed joint trajectories which could achieve the manipulation goal while satisfying stability constraints. Candidate plans were displayed in the Viewer as a sequence of ghosted renderings of the robot, through which the operator could "scrub." The operator could modify the plan before transmitting it to the robot for autonomous execution.

The operator initiated walking planning by specifying a navigation goal that triggered the footstep planner to compute a set of safe footsteps given the current estimate of the terrain. These footsteps were displayed in the Viewer for adjustment as needed. The walking planner then generated a whole-body plan and computed a stabilizing controller using a simple model of the robot. During each control step, the controller solved a quadratic program that minimized deviation from the desired dynamic walking plan while respecting the instantaneous dynamic, input, and friction constraints of the robot.

Team MIT's system also included a set of scripted recovery behaviors that were designed to return the robot to standing after a fall. If a successful recovery was deemed unlikely, the operator could switch the robot to a dynamic crawling mode that used a controller similar to the one used for walking.

The combination of walking and crawling planners and controllers allowed Team MIT to complete the course in all VRC walking trials. Recovery behaviors were successfully employed during the manipulation and driving tasks. The manipulation framework readily supported operations such as lifting a hose nozzle and turning a wall-mounted valve. It also allowed for precision alignment operations, such as inserting the nozzle into a standpipe. However, these required many attempts with small manual adjustments to the plan at each step. Finally, while the system was able to synthesize a plan for entering a vehicle, it was unable to execute this plan successfully. Instead, in the VRC driving tasks, a scripted jumping maneuver propelled the robot into the vehicle, but this resulted in body positions from which driving the vehicle was impossible.

Team MIT placed third out of twenty-two VRC teams. By finishing in the top six, Team MIT obtained an Atlas robot, and is now adapting its system for the DRC Trials in December of 2013.

\section{REFERENCES}

[1] "DARPA robotics challenge (DARPA-BAA-12-39)," Apr. 2012. [Online]. Available: https://www.fbo.gov/spg/ODA/DARPA/CMO/DARPABAA-12-39/listing.html

[2] T. Schneider and H. Schmidt, "Goby-acomms version 2: extensible marshalling, queuing, and link layer interfacing for acoustic telemetry," in 9th IFAC Conference on Manoeuvring and Control of Marine Craft, Arenzano, Italy, 2012. [Online]. Available: http://www.stoby.us/d1/mcmc2012_goby2.pdf 